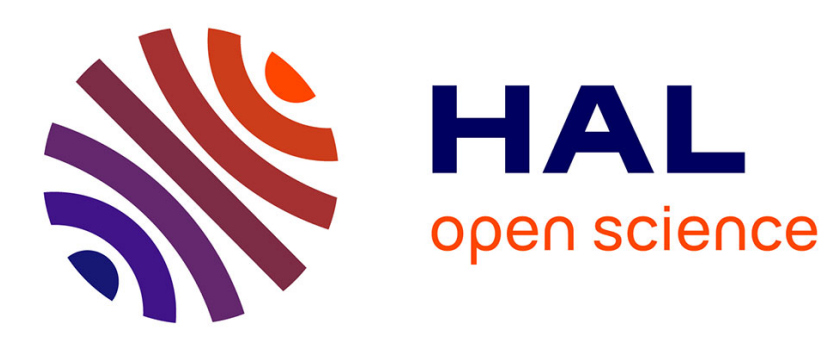

\title{
Low Complexity Adaptive Turbo Equalization for Multipath Underwater Communication
}

\author{
Zi Ye, Tarak Arbi, Benoit Geller
}

\section{To cite this version:}

Zi Ye, Tarak Arbi, Benoit Geller. Low Complexity Adaptive Turbo Equalization for Multipath Underwater Communication. OCEANS 2019 - Marseille, Jun 2019, Marseille, France. pp.1-5, 10.1109/OCEANSE.2019.8866873 . hal-02372263

\section{HAL Id: hal-02372263 https://hal.science/hal-02372263}

Submitted on 24 Nov 2021

HAL is a multi-disciplinary open access archive for the deposit and dissemination of scientific research documents, whether they are published or not. The documents may come from teaching and research institutions in France or abroad, or from public or private research centers.
L'archive ouverte pluridisciplinaire HAL, est destinée au dépôt et à la diffusion de documents scientifiques de niveau recherche, publiés ou non, émanant des établissements d'enseignement et de recherche français ou étrangers, des laboratoires publics ou privés. 


\title{
Low Complexity Adaptive Turbo Equalization for Multipath Underwater Communication
}

\author{
Zi Ye, Tarak Arbi, Benoit Geller \\ Department U2IS, ENSTA-ParisTech, Institut Polytechnique de Paris, France \\ \{zi.ye, tarak.arbi, benoit.geller\}@ensta-paristech.fr
}

\begin{abstract}
This paper deals with a receiver architecture where adaptive equalization and channel decoding are jointly performed in an iterative manner in order to both reduce intersymbol interference (ISI) and to recover phase error. To decrease the equalization complexity that can be prohibitive over some channels, we propose a low complexity turbo equalization with only a few coefficients to be updated. Simulation results show that our proposed low complexity equalizer allows a large reduction in computational complexity while also achieving better performance over multipath channels.
\end{abstract}

Index Terms-Turbo equalization, demodulation complexity, channel decoding, underwater acoustics communication.

\section{INTRODUCTION}

The underwater channel is one of the most challenging channels as the acoustic signal may suffer from both a very limited coherence bandwidth and from high Doppler effect. In particular the multipath propagation introduce ISI that can seriously limit the system performance. To work around this issue, several equalization algorithms have been studied in the literature [1-5]. Following the discovery of turbo decoding, in [1], a turbo equalization scheme has been proposed. Nevertheless, the complexity of such algorithm grows exponentially with the communication parameters, which prohibits its use in real life applications. To face this issue, numerous algorithms were proposed, such as adaptive turbo equalization [2] and linear filtering with decoding [3]. Those proposals allow a good BER performance with a reasonable complexity; in particular, to further reduce complexity over timevarying channels, [2] proposes to use some theoretical optimal equalizer taps expressions which depend on the estimated channel coefficients; however in practice, this may lead to suboptimal performances when the channel is poorly estimated [6]. Instead, in this paper, we propose to use a sparse adaptive turbo detector with only a few taps to be updated in order to lower down the complexity burden. Furthermore, the relative movement between the transmitter and the receiver or the swell may introduce some Doppler effect $[7,8]$ and may lead to poor equalization performance [4]. Thus, differently from [2], to enhance the BER performance, the proposed equalizer attempts to jointly cancel the ISI and to correct the phase error [9].

In recent years, numerous physical-layer algorithms and modulation schemes have been proposed. However, comparing

This work was sponsored by the Bridges project of the European H2020 program.

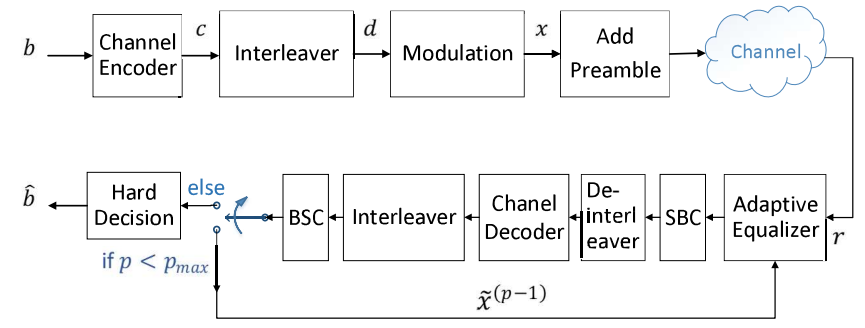

Fig. 1. System model

objectively these proposals in an undersea environment is often difficult because the simulation environments are different. In this paper, simulation results were obtained for both synthetic discrete time-varying channels and underwater simulation channels [10] labeled "WATERMARK"; this environment [10] includes a library of several impulse responses of 5 kinds of underwater channels measured in Norway, France and Hawaii and can be used on a Matlab platform.

The paper is organized as follows. Section II describes the system model. Section III presents the adaptive equalizer structure. Some numerical results obtained over real underwater channels and over discrete time-varying channels are displayed in section IV, as well as a detailed complexity analysis. At last, some conclusions are made in Section V.

\section{SySTEM MOdEL}

The transmission scheme is displayed in Fig. 1. The channel encoder is a systematic parallel convolutional turbo code of rate $1 / 3$ with generator polynomials equal to 5,7 . A Bit Interleaved Coded Modulation (BICM) system [11] is used to encode at rate $R_{c}$ the information bits $b$ at the transmitter side. To increase the diversity of the code, each set of $m=\log _{2} M$ interleaved coded bits $d$ are mapped into an M-QAM complex symbols $x$. It is worth mentioning that in practice a preamble, known by the receiver, should be added at the beginning of each frame. Indeed, this preamble is essential for the frame detection and the good convergence of the adaptive equalization $[2,10,12]$. Finally, those signals are transmitted through generally a time and frequency selective channel. This is the case for instance of the underwater channel as it can be observed in Fig. 2 which shows some impulse responses of a channel captured in a shallow stretch of Oslofjorden (NOF1) 

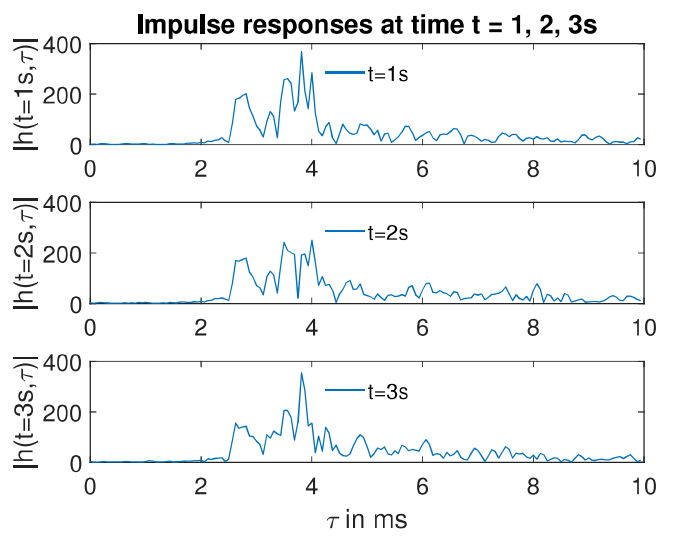

Fig. 2. Impulse responses at times $t=1,2,3 \mathrm{~s}$ of NOF1

TABLE I

THE MEASUREMENT PARAMETERS.

\begin{tabular}{|c|c|c|}
\hline Parameters & BCH1 & NOF1 \\
\hline Time of the year & May & June \\
\hline Water depth & $20 \mathrm{~m}$ & $80 \mathrm{~m}$ \\
\hline -3dB freq. band & $32.5-37.5 \mathrm{KHz}$ & $10-18 \mathrm{KHz}$ \\
\hline Duration & $59.4 \mathrm{~s}$ & $32.9 \mathrm{~s}$ \\
\hline Type & SIMO & SISO \\
\hline Hydrophones & 4 & 1 \\
\hline
\end{tabular}

over a distance of 750m [10] at different times. Furthermore, simulations of our proposal are performed as well in section IV over another submarine channel measured in the commercial harbour of Brest, France, noted BCH1. The measurement parameters of these two channels are summarized in Table I.

Similarly to [13], one can model the discrete received signal as

$$
r_{n}=\sum_{l} h_{l} x_{n-l}+w_{n},
$$

where $n$ refers to the time index, $w_{n}$ is the AWGN noise and $x_{n-l}$ (resp. $h_{l}$ ) indicates the transmitted symbols and the channel impulse response coefficients respectively.

At the receiver side, several signal processing algorithms are needed in order to correctly recover the transmitted frame. In this paper, we focus on the design of an algorithm performing jointly the equalization and the phase synchronization. As detailed in the next section, the phase estimation [14-18] is carried out separately from the equalization process as it evaluates more rapidly than the timing synchronization [1923] and this could be an additional difficulty if the equalization step had also to inherently follow those rapid variations.

\section{Adaptive Turbo Equalization}

\section{A. Adaptive equalizer structure}

As for the classical turbo equalizer [1], the receiver has to jointly perform equalization and channel decoding by the mean of exchanging extrinsic information. For each iteration $p$, the equalizer is fed in by received signal samples $R_{n}$ and estimated data $\bar{X}_{n}$ obtained from the previous iteration

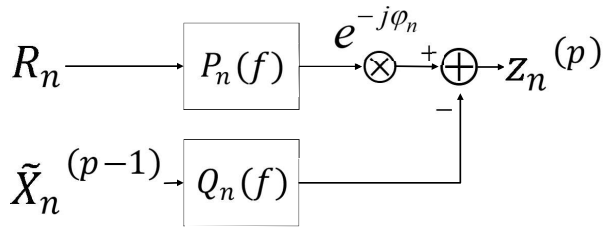

Fig. 3. Joint equalizer and phase estimator structure

$(p-1)$. Furthermore, Fig. 3 describes a joint equalizer and phase estimator structure, including two transversal filters $P_{n}(f)$ and $Q_{n}(f)$ respectively fed by the received symbols and by soft estimated ones at the previous iteration. The adaptive algorithm requires a training sequence known at the receiver side to initialise the equalizer coefficients and ensure convergence of the algorithm. Furthermore, during the tracking period, the coefficients are updated based on the decisions on estimated symbols $\bar{x}_{n}$ for all $p>1$.

In order to simplify the notation, the index $(p)$ is dropped in the sequel. The output of the equalizer can be expressed as:

$$
z_{n}=P_{n}^{T} R_{n} e^{-i \varphi_{n}}-Q_{n}^{T} \widetilde{X}_{n}
$$

where $\tilde{X}_{n}=\left[\tilde{x}_{n+L_{2}}, \cdots \tilde{x}_{n} \cdots, \tilde{x}_{n-L_{2}}\right]^{T}$ and $\tilde{x}_{n}$ can either be symbol $x_{n}$ during the preamble or estimated symbol $\bar{x}_{n}$ during the tracking period; $R_{n}=$ $\left[r_{n+L_{1}}, \cdots r_{n} \cdots, r_{n-L_{1}}\right]^{T}$ denotes the received symbols; $P_{n}=\left[p_{-L_{1}}(n), \cdots, p_{0}(n), \cdots, p_{L_{1}}(n)\right]^{T}$ and $Q_{n}=$ $\left[q_{-L_{2}}(n), \cdots, q_{0}(n)=0, \cdots, q_{L_{2}}(n)\right]^{T}$ are transversal filters of respective lengths $2 L_{1}+1$ and $2 L_{2}+1 ; \varphi_{n}$ is the estimated phase error. The coefficients of the filters are updated in order to minimize the estimated Mean-Square Error:

$$
M \hat{S} E(P, Q, \varphi)=E\left\{\left|z_{n}-\tilde{x}_{n}\right|^{2}\right\} .
$$

Similarly to [2, 4], the derivation of $M \hat{S} E$ with respect to $P$, $Q$ and $\varphi$ leads to the following gradients:

$$
\begin{cases}\nabla_{P}\left(\left|z_{n}-\tilde{x}_{n}\right|^{2}\right) & =2\left(z_{n}-\tilde{x}_{n}\right) R_{n}^{*} e^{i \varphi_{n}} \\ \nabla_{Q}\left(\left|z_{n}-\tilde{x}_{n}\right|^{2}\right) & =-2\left(z_{n}-\tilde{x}_{n}\right) \widetilde{X}_{n}^{*} \\ \nabla_{\varphi}\left(\left|z_{n}-\tilde{x}_{n}\right|^{2}\right) & =2 \operatorname{Im}\left(P_{n}^{T} R_{n} e^{-i \varphi_{n}}\left(z_{n}-\tilde{x}_{n}\right)^{*}\right) .\end{cases}
$$

We obtain the corresponding update coefficients expressed as:

$$
\begin{aligned}
P_{n+1} & =P_{n}-\mu\left(z_{n}-\tilde{x}_{n}\right) R_{n}^{*} e^{i \varphi_{n}} \\
Q_{n+1} & =Q_{n}+\mu\left(z_{n}-\tilde{x}_{n}\right) \widetilde{X}_{n}^{*} \\
\varphi_{n+1} & =\varphi_{n}-\gamma \operatorname{Im}\left(P_{n}^{T} R_{n} e^{-i \varphi_{n}}\left(z_{n}-\tilde{x}_{n}\right)^{*}\right),
\end{aligned}
$$

where $\mu$ and $\gamma$ are the appropriate step sizes. It is also worth to note that the channel response of the underwater channel can be particularly large $[4,24,25]$; thus, in order to have an efficient equalization, $L_{1}$ and $L_{2}$ should also be large enough, leading to a significant impact on the receiver architecture.

Consequently, once the convergence during the preamble is established, we propose to put the coefficients of the filters that are below a certain threshold to zero. Similarly, one can keep a certain predefined percentage of the filters coefficients 
with the highest energy and set the others to zero. The new sparse transversal $[24,26]$ filters are labeled in the sequel as $P_{n}^{\prime}(f)$ and $Q_{n}^{\prime}(f)$.

\section{B. Symbol to Bit Converter (SBC)}

The role of the SBC (see Fig. 1) is to compute the LLR value of the coded bits from the equalized symbol $z_{n}$ which will be transmitted to the soft-input soft-output channel decoder. $z_{n}$ can be decomposed as the sum of two parts [5]:

$$
z_{n}=\beta_{0} \tilde{x}_{n}+\nu_{n}
$$

where $\beta_{0} \tilde{x}_{n}$ is the desired signal with a constant bias factor $\beta_{0}, \nu_{n}$ is the sum of the noise and residual interference at the output of the equalizer. It can be found, assuming a Gaussian distribution of the residual ISI, that $\nu_{n}$ follows a complex Gaussian distribution with zero mean and total variance $\sigma_{v}^{2}=\sigma_{\tilde{x}}^{2} \beta_{0}\left(1-\beta_{0}\right)$, where $0 \leq \beta_{0} \leq 1$ and $\sigma_{\tilde{x}}^{2}$ is the signal $\tilde{x}$ variance. The extrinsic LLRs of the mapped bits $l^{i}(x)\left(i=0,1, \ldots, \log _{2} M-1\right)$ are given by:

$$
L L R\left(l^{i}(x)\right)=\ln \frac{\sum_{s \in \boldsymbol{\chi}_{b}^{1}} \exp \left(-\frac{\left|z_{n}-\beta_{0} s\right|^{2}}{\sigma_{v}^{2}}\right)}{\sum_{s \in \boldsymbol{\chi}_{b}^{0}} \exp \left(-\frac{\left|z_{n}-\beta_{0} s\right|^{2}}{\sigma_{v}^{2}}\right)},
$$

where $l^{i}(x)$ is the $\mathrm{i}$-th bit of symbol $x$ and $\chi_{b}^{i}$ denotes the set of the constellation points whose i-th bit is $b=1$ or 0 .

\section{Bit to Symbol Converter (BSC)}

For all $p>1$, one calculates the estimated value $\bar{x}_{n}^{(p-1)}$ from the BSC using the output LLR values of the channel decoder at the iteration $p-1$. The soft estimation $\bar{x}_{n}$ is given by:

$$
\bar{x}_{n}^{(p-1)}=\sum_{s \in \mathbf{X}} s\left(\prod_{i=1}^{\log _{2} M} \operatorname{Pr}\left(l^{i}\left(x_{n}\right)=l^{i}(s)\right)\right),
$$

where $\mathbf{X}$ refers to the signal constellation set. The term $\operatorname{Pr}\left(l^{i}\left(x_{n}\right)=j\right)$ where $j$ can either be 0 or 1 can be written as:

$$
\begin{aligned}
& \operatorname{Pr}\left(l^{i}\left(x_{n}\right)=1\right)=\frac{\exp \left(\operatorname{LLR}\left(l^{i}\left(x_{n}\right)\right)\right)}{1+\exp \left(\operatorname{LLR}\left(l^{i}\left(x_{n}\right)\right)\right)} \\
& \operatorname{Pr}\left(l^{i}\left(x_{n}\right)=0\right)=1-\operatorname{Pr}\left(l^{i}\left(x_{n}\right)=1\right) .
\end{aligned}
$$

\section{NUMERICAL RESULTS}

\section{A. Complexity Analysis}

The detailed analysis on the complexity of the algorithm is evaluated in terms of Real Multiplication (RM) and Real Sum (RS), where a RS can either be a real addition or a real subtraction.

- In equation (2) : Multiplying the $P_{n}$ vector by $R_{n}$ requires $3\left(2 L_{1}+1\right)+4 L_{1} \mathrm{RSs}$ and $4\left(2 L_{1}+1\right) \mathrm{RMs}$, then multiplying the term $\left(P_{n}^{T} R_{n}\right)$ by $e^{-i \varphi_{n}}$ needs $3 \mathrm{RSs}$ and 4RMs. Similarly, multiplying the $Q_{n}$ vector by $\widetilde{X}_{n}$ requires $3\left(2 L_{2}+1\right)+4 L_{2} \mathrm{RSs}$ and $4\left(2 L_{2}+1\right)$ RMs.
TABLE II

COMPLEXITY COMPARISON BETWEEN THE PROPOSED SPARSE ADAPTIVE AND THE FULL COMPLEXITY EQUALIZERS

\begin{tabular}{|c|c|c|}
\hline & Real Multiplications & Real Sums \\
\hline & $\left(16 L_{1}+16 L_{2}+31\right) N_{p}$ & $\left(20 L_{1}+20 L_{2}+28\right) N_{p}$ \\
A & $+\left(16 L_{1}+16 L_{2}+31\right) p_{\max }\left(N_{s}-N_{p}\right)$ & $+\left(20 L_{1}+20 L_{2}+28\right) p_{\max }\left(N_{s}-N_{p}\right)$ \\
& $+2 \mathrm{MlogM}\left(p_{\max }-1\right) N_{s}$ & $+2 \mathrm{M}\left(p_{\max }-1\right) N_{s}$ \\
\hline & $\left(16 L_{1}+16 L_{2}+31\right) N_{p}+$ & $\left(20 L_{1}+20 L_{2}+28\right) N_{p}+$ \\
B & $\left(16 L_{1}^{\prime}+16 L_{2}^{\prime}+31\right) p_{\max }\left(N_{s}-N_{p}\right)$ & $\left(20 L_{1}^{\prime}+20 L_{2}^{\prime}+28\right) p_{\max }\left(N_{s}-N_{p}\right)$ \\
& $+2 \mathrm{MlogM}\left(p_{\max }-1\right) N_{s}$ & $+2 \mathrm{M}\left(p_{\max }-1\right) N_{s}$ \\
\hline
\end{tabular}

Therefore, equation (2) takes a total of $10 L_{1}+10 L_{2}+11$ RSs and $8 L_{1}+8 L_{2}+12$ RMs.

- In equation (5): Computing $\mu\left(z_{n}-\tilde{x}_{n}\right)$ requires $2 \mathrm{RSs}$ and 2RMs, multiplying it with $e^{-i \varphi_{n}}$ needs 3RSs and 4RMs. Multiplying the previous product by $R_{n}^{*}$ requires $3\left(2 L_{1}+1\right)$ RSs and $4\left(2 L_{1}+1\right)$ RMs. Thus, equation (5) needs globally $10 L_{1}+8 \mathrm{RSs}$ and $8 L_{1}+10$ RMs.

- In equation (6): The multiplication with $\widetilde{X}_{n}^{*}$ requires $3\left(2 L_{2}+1\right)$ RSs and $4\left(2 L_{2}+1\right)$ RMs. Thus, equation (6) requires a total of $10 L_{2}+5 \mathrm{RSs}$ and $8 L_{2}+4 \mathrm{RMs}$.

- In equation (7): It takes $3 \mathrm{RSs}$ and 4RMs to multiply with $\left(z_{n}-\tilde{x}_{n}\right)^{*}$. Thus, equation (7) totally requires $4 \mathrm{RSs}$ and 5RMs.

- In equation (10): The product of the term $\operatorname{Pr}\left(l^{i}\left(x_{n}\right)=l^{i}(s)\right)$ needs $2 M \log _{2} M$ RMs. Thus, equation (10) requires a total amount of $2 M \mathrm{RSs}$ and $2 M \log _{2} M$ RMs.

Therefore, performing the full complexity equalization of a symbol globally requires $\left(20 L_{1}+20 L_{2}+28+2 M\right)$ RSs and $\left(16 L_{1}+16 L_{2}+31+2 M \log _{2} M\right) \quad$ RMs, while performing the sparse proposal of a symbol in tracking period requires $\left(20 L_{1}^{\prime}+20 L_{2}^{\prime}+28+2 M\right)$ RSs and $\left(16 L_{1}^{\prime}+16 L_{2}^{\prime}+31+2 M \log _{2} M\right) \quad$ RMs, where $\left(2 L_{1}^{\prime}\left(\right.\right.$ resp. $\left.\left.L_{2}^{\prime}\right)+1\right)$ is the number of non-zero coefficients of the filter $P_{n}^{\prime}(f)$ (resp. $\left.Q_{n}^{\prime}(f)\right)$.

Table II compares the complexity between the full complexity equalizer and the sparse proposal (referred as A and $\mathrm{B}$ respectively) with $p_{\max }$ iterations and $N_{s}$ (resp. $N_{p}$ ) corresponds to the number of total symbols (resp. preamble).

\section{B. Time-varying Channels}

In addition to the WATERMARK real recorded channels, we also perform simulations on a synthetic channel. As in [2], in this part, channel coefficients are modeled as:

$h_{l}(n)=\sqrt{\frac{P_{l}}{I}} \sum_{i=1}^{I} e^{j\left(2 \pi\left(f_{d} \cos \xi_{l, i}\right) n T+\psi_{l, i}\right)}, l=0,1, \cdots, L-1$.

where at time $\mathrm{n}, P_{l}$ is the mean power of the $l-t h$ channel path, random variables $\xi_{l, i}$ and $\psi_{l, i}$ obey uniform distributions over the interval $[0,2 \pi]$ and $I$ is fixed to 10 in our simulations. Generally the Doppler effect is characterised by $B_{d} T$ and $B_{d}=2 f_{d}$ refers to the Doppler band.

For simulations, the information data were encoded by a $1 / 3$ rate turbo code, a training sequence made up of 25 symbols, 


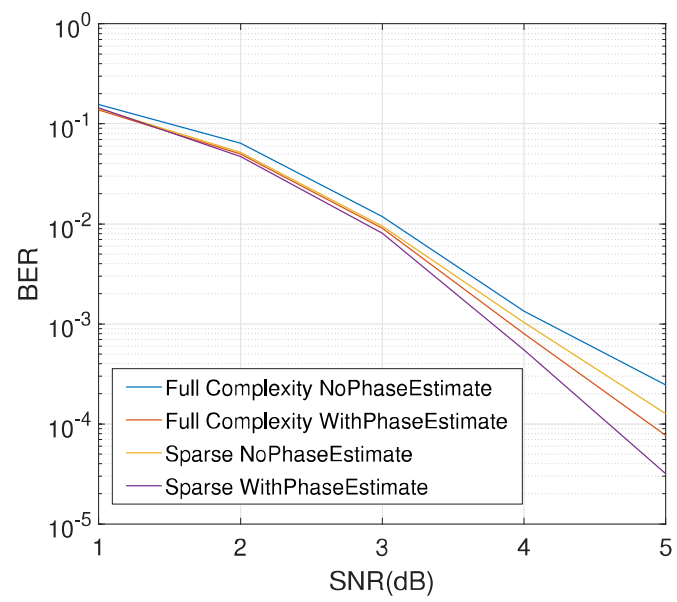

Fig. 4. QPSK BER comparison between the full complexity equalizer and the proposed one, with and without phase synchronization over the Rayleigh multipath channel

out of a total length of 125 symbols per packet, is transmitted to make the equalizer converge. The number of channel taps in (12) $L$ is equal to 5 . All packets are successively transmitted over the continuously varying Rayleigh multipath channel (12). At the first iteration, the number of taps for $P_{n}(f)$ and $Q_{n}(f)$ are equal to 21 , the central coefficient of $P_{n}(f)$ is set to 1 ; in the training (resp. tracking) period $\mu$ is equal to 0.001 (resp. 0.0003), $\gamma$ is set to 0.01 (resp.0.006). For following iterations, the number of taps of $P_{n}(f)$ and $Q_{n}(f)$ are equal to 21 and 31; in the training (resp. tracking) period $\mu$ is equal to 0.001 (resp. 0.0002), $\gamma$ is set to 0.01 (resp.0.006). The number of turbo equalization is $p_{\max }=5$. In the tracking period, the sparse equalizer turns off 14 (resp. 22) among the 21 (resp. 31) coefficients of $P_{n}(f)$ (resp. $Q_{n}(f)$ ). Thus, the number of nonzero coefficients $P_{n}^{\prime}(f)$ and $Q_{n}^{\prime}(f)$ are 7 and 9 respectively.

Fig. 4 compares the BER performance between the full complexity algorithm and the sparse proposal, with and without phase synchronization respectively, for the QPSK constellation over the Rayleigh multipath channel. It can be observed that our sparse proposal allows a BER performance improvement of about $0.3 \mathrm{~dB}$ compared with the full complexity algorithm, and in addition, performing phase synchronization separately with equation (7) allows an additional gain of $0.5 \mathrm{~dB}$. Furthermore, with table II, we found that compared with the full complexity equalizer, the amount of real multiplications (resp. real sums) of our sparse equalizer is reduced by $59 \%$ (resp. 60\%).

\section{WATERMARK}

To obtain Bit Error Rate (BER) curves over the underwater channel, user frames of 2048 bits are coded with a turbo code of rate 1/3. A training sequence made of 511 symbols is added before each user frame for both frame detection and convergence of the equalizer. The information is transmitted at a bit rate of roughly $2 \mathrm{kbit} / \mathrm{s}$ (QPSK) and $4 \mathrm{kbit} / \mathrm{s}$ (16-QAM) at a $14 \mathrm{kHz}($ resp. $35 \mathrm{kHz})$ carrier frequency over the channel

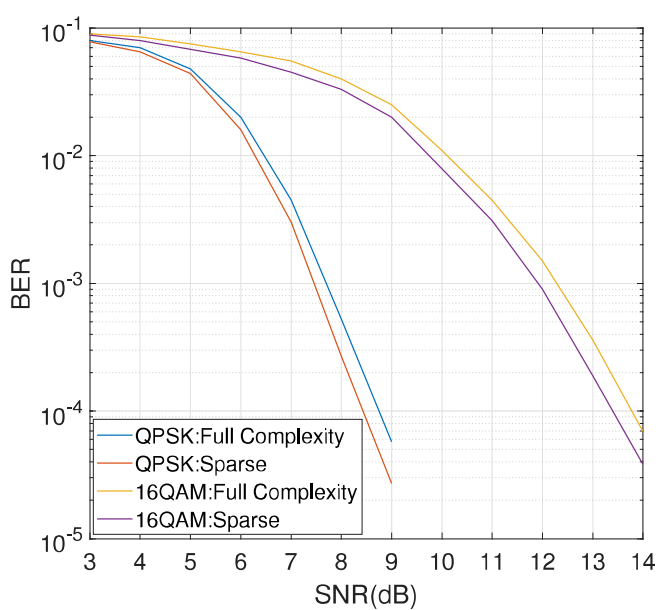

Fig. 5. BER comparison between the full complexity equalizer and the proposed one over the channel NOF1

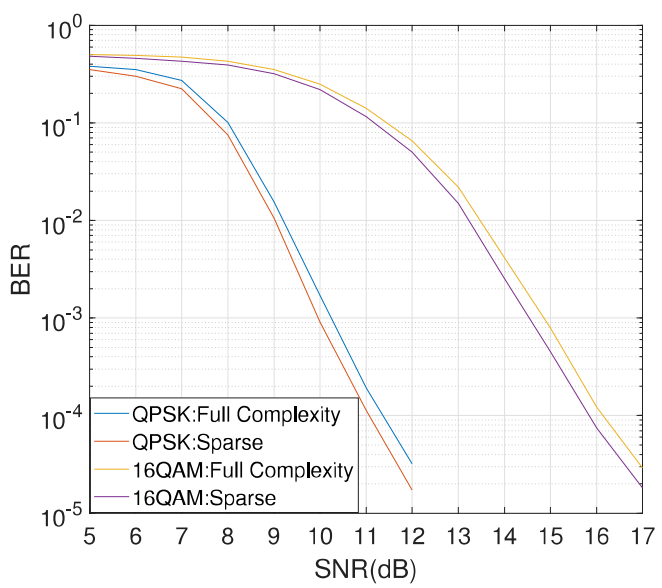

Fig. 6. BER comparison between the full complexity equalizer and the proposed one over the channel $\mathrm{BCH} 1$

NOF1 (resp. BCH1). At the first iteration, the number of taps for $P_{n}(f)$ and $Q_{n}(f)$ are equal to 41 and 51 respectively, the central coefficient of $P_{n}(f)$ is set to $1 ; \gamma$ is set to 0.08 . In the training (resp. tracking) period $\mu$ is equal to 0.003 (resp. 0.0005 ). The number of turbo equalization is $p_{\max }=10$. In the tracking period, the sparse equalizer turns off 30 (resp. 38) among the 41 (resp. 51) coefficients of $P_{n}(f)$ (resp. $Q_{n}(f)$ ).

Fig. 5 (resp. Fig. 6) compares the BER performance between the full complexity algorithm and the sparse proposal for the QPSK and 16-QAM constellations over the channel NOF1 (resp. BCH1). It can be observed that our sparse proposal allows a BER performance improvement of about 0.4 (resp. 0.3) dB. This is because small coefficients of the full complexity equalizer add supplemental unwanted noise to $z_{n}$ and are weakly related to the channel. Furthermore, with table II, we found that compared with the full complexity equalizer, the amount of real multiplications (resp. real sums) of our sparse equalizer is reduced by $70 \%$ (resp. $71 \%$ ). 


\section{CONCLUSION}

In this paper, a fully adaptive low complexity joint iterative channel equalization and channel decoding process was proposed. Indeed, for our proposal, only a small number of coefficient updates make the low complexity equalizer suited for real-time processing. The equalizer was successfully tested over Rayleigh time-varying and over recorded multipath underwater channels. The good performance and the simplicity of our proposal make it very attractive for practical implementation. Finally, unsuited step sizes can have serious impacts on the algorithm, thus, in the future, we want to adjust our proposal so as to adaptively update these step sizes. We would also like to test several channel coding schemes such as block turbo codes [27-29], coded modulation [30-32] with larger coding rates in order to save bandwidth [33] without sacrificing the efficiency of our equalizing proposal.

\section{ACKNOWLEDGEMENT}

The authors want to thank F.X. Socheleau for his instructive advice and useful suggestions. We are grateful for his help in the completion of this paper.

\section{REFERENCES}

[1] C. Douillard, M. Jezequel, C. Berrou, A. Picart, P. Didier, "Iterative correction of intersymbol interference: turbo-equalization", European Transactions on Telecommunications, Wiley, 1995, 6 (5), pp. 507-512.

[2] C. Laot, A. Glavieux and J. Labat, "Turbo Equalization: Adaptive Equalization and Channel Decoding Jointly Optimized," IEEE Journal on Selected Areas in Communications, vol. 19, no. 9, Sep 2001.

[3] M. Tuchler and A. C. Singer, "Turbo Equalization: An Overview," IEEE Trans. on Information Theory, vol. 57, no. 2, pp. 920-952, Feb 2011.

[4] B. Geller, V. Capellano, J.M. Brossier, A. Essebbar, G. Jourdain, "Equalizer for Video Rate Transmission in Multipath Underwater Communications," IEEE Journal of Oceanic Engineering, vol. 21, no. 2, Apr 1996.

[5] C. Laot and R. L. Bidan, "Adaptive MMSE turbo equalization with highorder modulations and spatial diversity applied to underwater acoustic communications," 17th European Wireless 2011 - Sustainable Wireless Technologies, Vienna, Austria, 2011.

[6] Y. Zhang, Y. V. Zakharov, J. Li, "Soft-Decision-Driven Sparse Channel Estimation and Turbo Equalization for MIMO Underwater Acoustic Communications”, IEEE Access, vol. 6, pp. 4955-4973, Jan 2018.

[7] J. Li, Y. Zakharov,B. Henson, "Multibranch autocorrelation method for Doppler Estimation in Underwater Acoustic Channels," IEEE Journal of oceanic engineering, vol. 43(4), pp. 1099-1113, Oct 2018.

[8] R. Diamant, A. Feuer, L. Lampe, "Choosing the right signal: Doppler shift estimation for underwater acoustic signals," Proc. of the 7th ACM Int. Conf. On Underwater Networks and Systems, Los Angeles, Nov 2012.

[9] J. Yang and B. Geller, "Near-optimum Low-Complexity Smoothing Loops for Dynamical Phase Estimation", IEEE Transactions on Signal Processing, vol. 57, no. 9, pp. 3704-3711, Sept 2009.

[10] P.A. van Walree, F.X. Socheleau, R. Otnes, and T. Jenserud, "The Watermark Benchmark for Underwater Acoustic Modulation Schemes," IEEE Journal of Oceanic Engineering, pp. 1007-1018 vol. 42, Oct 2017.

[11] L. Szczecinski and A. Alvarado, "Bit-Interleaved Coded Modulation: Fundamentals, Analysis and Design," John Wiley and sons, UK, 2015.

[12] T. Arbi, B. Geller, "Multi-standard Receiver for Medical IoT Sensor Networks" in Challenges of the internet of things : technique, use, ethics, Hoboken, NJ : Wiley-ISTE, October 2018, p.105-121.

[13] Z. Ye, T. Arbi, F.X. Socheleau, B. Geller, "Fast Soft Demapping for Underwater Acoustic Communications With Signal Space Diversity," IEEE Oceans, 2018. Oct 2018, Charleston, United States.

[14] J.M. Brossier, P.O. Amblard, B. Geller, "Self adaptative PLL for General QAM Constellations," Proceedings of EUSIPCO, pp 631-635 Toulouse, Sept 2002.
[15] J. Yang, B. Geller, C. Herzet, J.M. Brossier, "Smoothing PLLs for QAM Dynamical Phase Estimation", Proceedings of IEEE International Conference on Communications, ICC'09, Dresden, June 2009.

[16] J. Yang, B. Geller, A. Wei, "Approximate Expressions for Cramer-Rao Bounds of Code Aided QAM Dynamical Phase Estimation," Proceedings of IEEE International Conference on Communications, ICC'09, Dresden, June 2009.

[17] S. Bay, B. Geller, A. Renaux, J.P. Barbot, J.M. Brossier, "On the Hybrid Cramer-Rao Bound and Its Application to Dynamical Phase Estimation," IEEE signal processing letters vol. 15, pp. 453-456, 2008.

[18] J. Yang, B. Geller, S. Bay, "Bayesian and Hybrid Cramer-Rao Bounds for the Carrier Recovery Under Dynamic Phase Uncertain Channels,' IEEE Transactions on Signal Processing, vol. 59 (2), pp. 667-680, Feb. 2011.

[19] I. Nasr, B. Geller, L.N. Atallah, S. Cherif, "Performance study of a near maximum likelihood code-aided timing recovery technique", IEEE Transactions on Signal Processing, vol. 64, pp. 799-811, Feb 2016.

[20] I. Nasr, L.N. Atallah, S. Cherif, B. Geller, "Near MAP Dynamical Delay Estimator and Bayesian CRB for Coded QAM Signals," IEEE Transactions on Wireless Communications, vol. 17 (1), pp. 636-651, Jan 2018

[21] I. Nasr, L.N. Atallah, B. Geller, S. Cherif, "CRB derivation and new code-aided timing recovery technique for QAM modulated signals," Proc. of IEEE International Conference on Communications (ICC), pp. 4901-4906, London, June 2015.

[22] U. Mengali and A.N. D'Andrea, "Synchronization Techniques for Digital Receivers," Plenum Press, 1997.

[23] F. Gardner, "Demodulator reference recovery techniques suited for digital implementation," 1988, available on-line.

[24] B. Geller, J.M. Broissier, V. Capellano, "Equalizer for high data rate transmission in underwater communications," Proc. of OCEANS'94, vol. 1, Oceans Engineering for Today's Technology and Tomorrow's preservation, Brest, Sept 1994.

[25] B. Geller, V. Capellano, G. Jourdain, "Equalizer for real time high rate transmission in underwater communications," Proc. of ICASSP-95, pp. 302-305, Detroit, May 1995.

[26] M. Kocic, D. Brady, M. Stojanovic. "Sparse equalization for real-time digital underwater acoustic communications," Proc. IEEE Oceans, vol.1, pp. 1417-1422, San Diego, Oct. 1995.

[27] I. Diatta, D. De Geest, B. Geller, "Reed Solomon turbo codes for high data rate transmission" Proc. of Vehicular Technology Conference, 2004. VTC 2004-Spring, pp. 1023-1027, Milan, May 2004.

[28] B. Geller, I. Diatta, J.P. Barbot, C. Vanstraceele, F. Rambeau, "Block turbo codes: From architecture to application," Proc. of ISIT, IEEE International Symposium on Information Theory, 2006, pp. 1813-1816, Seattle, July 2006.

[29] C. Vanstraceele, B. Geller, J.P. Barbot, J.M. Brossier, "A Low Complexity Block Turbo Decoder Architecture," IEEE Transactions on Communications, vol. 56 (12), pp.1985-1989, Dec 2008.

[30] S. Lin and D. J. Costello, "Error Control Coding Prentice Hall, 2nd ed. 2004.

[31] J. Yang, K. Wan, B. Geller, C.A. Nour, O. Rioul, C. Douillard, "A lowcomplexity 2D signal space diversity solution for future broadcasting systems," Proc. of IEEE International Conference on Communications (ICC), pp. 2762-2767 London, June 2015.

[32] T. Arbi, B. Geller, J. Yang, C. A. Nour, O. Rioul, "Uniformly Projected RCQD QAM: A Low-Complexity Signal Space Diversity Solution Over Fading Channels With or Without Erasures," IEEE Transactions on Broadcasting, vol. 64, no 4, pp. 893-815, Dec 2018.

[33] R. Diamant, L. Lampe, "Adaptive error-correction coding scheme for underwater acoustic communication networks," IEEE Journal of Oceanic Engineering, vol. 40 (1), pp. 104-114, Jan 2015. 